\title{
CIST
}

\section{GATEWAY ARCHITECTURES FOR HOME CARE APPLICATIONS USING WIRELESS SENSOR NETWORKS}

\author{
Axel Sikora ${ }^{1)}$, Nathan Braun ${ }^{2)}$, Steffen Jaeckel ${ }^{2)}$, Daniel Jaeckle ${ }^{2)}$ \\ 1) University of Applied Sciences Offenburg, Badstrasse 24, D77652 Offenburg, Germany \\ axel.sikora@hs-offenburg.de, www.hs-offenburg.de \\ 2) Steinbeis Innovation Center Embedded Design and Networking, Poststrasse 35, D79423 Heitersheim, Germany \\ \{nathan.braun; steffen.jaeckel; daniel.jaeckle\}@stzedn.de, www.stzedn.de
}

\begin{abstract}
Home Care Applications and Ambient Assisted Living become increasingly attractive. This is caused as well by market pull, as the number of elderly people grows monotonously in highly-developed countries, as well as by technology push, as technological advances and attractive products pave the way to economically advantageous offerings. However, a significant number of challenges remain for real-life applications. Those include the lack of sufficiently standardized and interoperable solutions and thus, the necessity of gateways for integrated solutions, restrictions of the energy budgets, and scalability of solutions with regard to cost and network size.

This paper presents the experience from the inCASA project (Integrated Network for Completely Assisted Senior Citizen's Autonomy), where architectures for heterogeneous physical and logical communication flow are examined.
\end{abstract}

Keywords: wireless networking, home care, ambient assisted living, Continua, ZigBee, Bluetooth

\section{INTRODUCTION}

The number of elderly people across Europe, from now to year 2030, will unbelievably rise, and they are growing more healthy generation-bygeneration. Elderly people in good health conditions live longer, but there are a few factors that could radically impact on elderly person's quality of life and reducing his life expectation:

- psycho-emotional instability, related to the patient mental condition;

- the loneliness, related to the user environmental conditions and social life;

- illness, the patient health status.

Many products and solutions are already available in the market [1] and cover both telecare and telehealth applications. Telecare includes social supervision, such as home alarm [3], while telehealth applications have much stronger medical aspects, such as telecardiology [2]. In addition, a broad variety of scientific activities is ongoing in this field. However, it can be observed that most of the current solutions are still isolated devices with no or few interfaces to the outside world. Some newer devices allow networking capabilities, but by far too often - with the use of proprietary or closed communication protocols.

In order to overcome these shortcomings, the aim of objective 1.3 of the PSP programme of the
European Commission is to "significantly improve quality of life and social care for the ageing population" and "substantially prolong the time that elderly people can live independently at home”, an enormous number of national and European projects are on their way. The inCASA project (Integrated Network for Completely Assisted Senior Citizen's Autonomy) [4] is one of those and aims at developing a system that will support the aging population and facilitate them to feel well in their own homes. The proposed application will monitor biometric data and track environment parameters of elderly users into their own home.

The remainder of the paper is organized as follows. In ch. 2, possible system architectures and their elements are discussed with its advantages and disadvantages, whereas ch. 3 explains the proposed solution. Then, ch. 4 will give an outlook on the pilot installations, which are currently equipped with the described devices.

\section{SYSTEM ARCHITECTURE}

\subsection{OVERALL NETWORK ARCHITECTURE}

For both telecare and telehealth applications, the overall network architecture can be described with four communication levels, as they are shown in 
Fig. 1. This classification is very similar to other network topologies with widely distributed sensor

and actuator nodes, such as automatic meter reading [6].

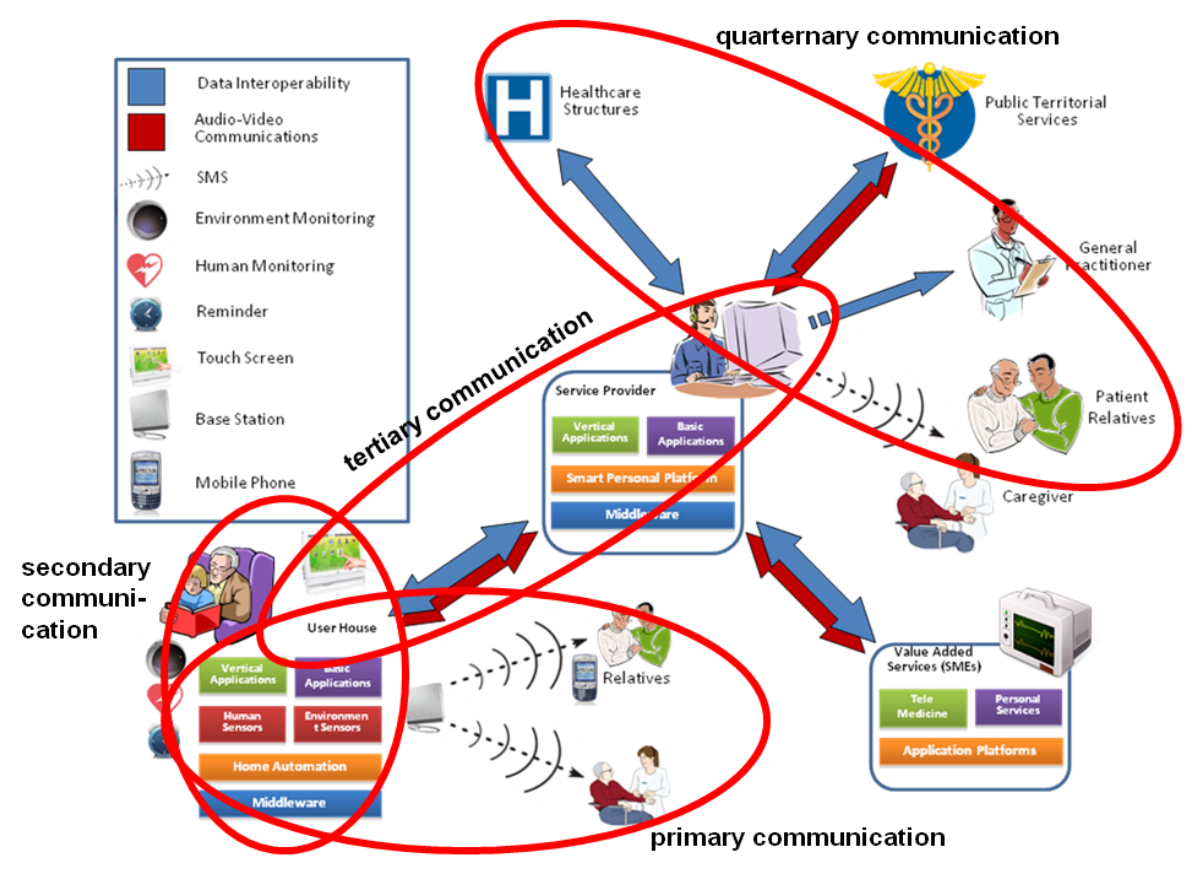

Fig. 1 - Overall Four-Layer Network Architecture

The primary communication covers the path between a local sensor or actuator and a data collector or gateway. Unfortunately, today's world of primary communication is still very heterogeneous with several dozens of technical alternatives [5].

The secondary communication provides the data back to a display at the customer's premises. There are lots of discussions about the best alternative for this secondary communication.

- One alternative is the direct communication from the end-user device to the gateway or middleware.

- The other alternative is the provisioning of the data from the backend system.

It should be mentioned that these two options are often presented as alternatives. However, it is well possible to combine both approaches.

The tertiary communication connects the data collector or gateway to the provider of the functionality (utility). In general, this part of the communication uses public infrastructure. Of course, the Internet protocol is the most widely used network protocol in this field. From data transport point of view, mostly transparent channels over circuit or packet switched networks are used, i.e. ISDN, GPRS, ATM/DSL. In case of legacy telemonitoring systems, the sensors are directly hooked up to the backend system via what is now called tertiary communication.

The quarternary communication connects various backend systems.

In the authors' part of the project, the major focus is on the primary and tertiary communication solutions. Thus, also this paper restricts to it.

\subsection{LEGACY NETWORK PROTOCOLS AND DATA MODELS}

There is a large variety of different network protocols and data models, which are used for home care applications. Historically, there are two approaches, which impede interoperability: In the worst case, the data models and the network protocols are completely proprietary and not opened to the public. In the better case, they are public, but linked to a certain network protocol. This is the case for protocols like ZigBee, Bluetooth or EnOcean Radio Protocol (ERP), which define their specific application profiles. In these cases, interconnection implies a gateway covering the complete communication stacks from layer 1 through to layer 7.

\subsection{CONTINUA AND IEEE11073}

Fortunately, this legacy approach is overcome by the application profiles from Continua Alliance [7]. It is specified in IEEE11073 and contains the overall architecture and specific devices. The basic network management is described in the Optimized Exchange Protocol (IEEE 11073-20601), which includes three major elements: The Domain Information Model (DIM), the Service Model (SM), and the Communications Model (CM). In addition, it contains various data transport models, e.g. 
IEEE11073-10404 for Pulse Oximeter, IEEE11073-10406 for Pulse / Heart Rate, etc.

IEEE 11073-20601 defines the exchange protocol for information between the agent (sensor) and manager, mostly denominated as Application Hosting Device (AHD). It is designed to be extensible and flexible, and plug and play. To accomplish, it uses object descriptions of the device and data sources. The protocol supports self description of the object model on association.

The major benefit of these profiles is their independence from lower layer protocols, i.e. Continua profile are taken over in Bluetooth Health Device Profile (HDP) [8] and in ZigBee Health Care (ZHC) [9] Profile. It is also applicable over IP and Glowpan.

However, it also should be mentioned that these profiles are still quite new and thus are not reflected in all related sub-standards. I.e. HDP is not integrated into Bluetooth Ultra Low Energy (ULE) at the time of writing this paper.

\subsection{ANTICIPATED SENSOR INTERFACES}

In the inCASA project, a broad variety of sensors shall be integrated into the system. These combine health related, home automation, and ambience sensors. I.e. it is anticipated to measure blood pressure, blood oxygen level, body temperature, blood sugar, heart frequency, partial prothrombin time/INR, weight, symptoms scoring, peritoneal dialysis, physiological rehab (back/knee), movement at home, contact (e.g. door/bed/chair), wrist movement (actigraphy), ambient temperature, ambient humidity, water leaks, gas leaks.

\section{PROPOSED SOLUTION}

\subsection{MIDDLEWARE}

Despite all the activities for homogenization and standardization, it is clear that not all applications, markets and market players follow these specifications immediately. Therefore, it is obvious that some meta-level is required to integrate all these sensors and data models into one system, although the mid-term future will strive for interoperable solutions, as they are described in [16].

In large scale server system, this integration is supported by a middleware software, which allows multiple processes running on one or more machines to interact. For heterogeneous distributed embedded systems, the same idea has been realized in the Hydra middleware approach (Networked Embedded System Middleware for Heterogeneous Physical Devices in a Distributed Architecture) [10], which is an open source European project. Recently, Hydra was renamed to The LinkSmart middleware is since some time available for download as Open Source from SourceForge and will be maintained by the LinkSmart Foundation [15].

\subsection{MULTI-LEVEL NETWORKS}

Although the Hydra platform is optimized for resource efficiency, it still comes with a lot of different elements and, consequently, a significant complexity (cf. Fig 2). As a result, in most cases an (embedded) PC-hardware is utilized. Therefore, during the course of the inCASA project, a second level of gateway was introduced, which serves as a low cost, low complexity, and low energy gateway, and is described in section $\mathrm{C}$.

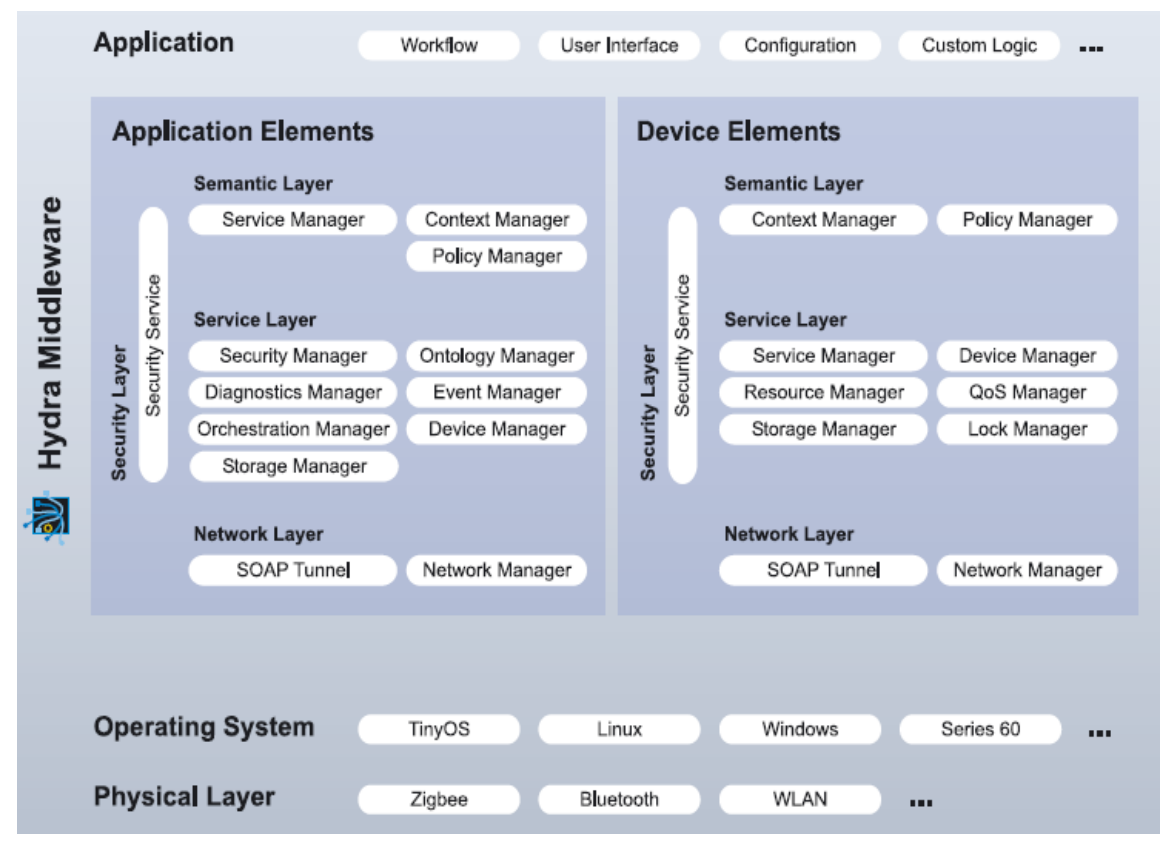

Fig. 2 - Elements of the Hydra Middleware platform [10] 
The resulting network architecture is shown in Fig. 3. It is currently under development and will be

applied in various pilot installations (cf. ch. 4).

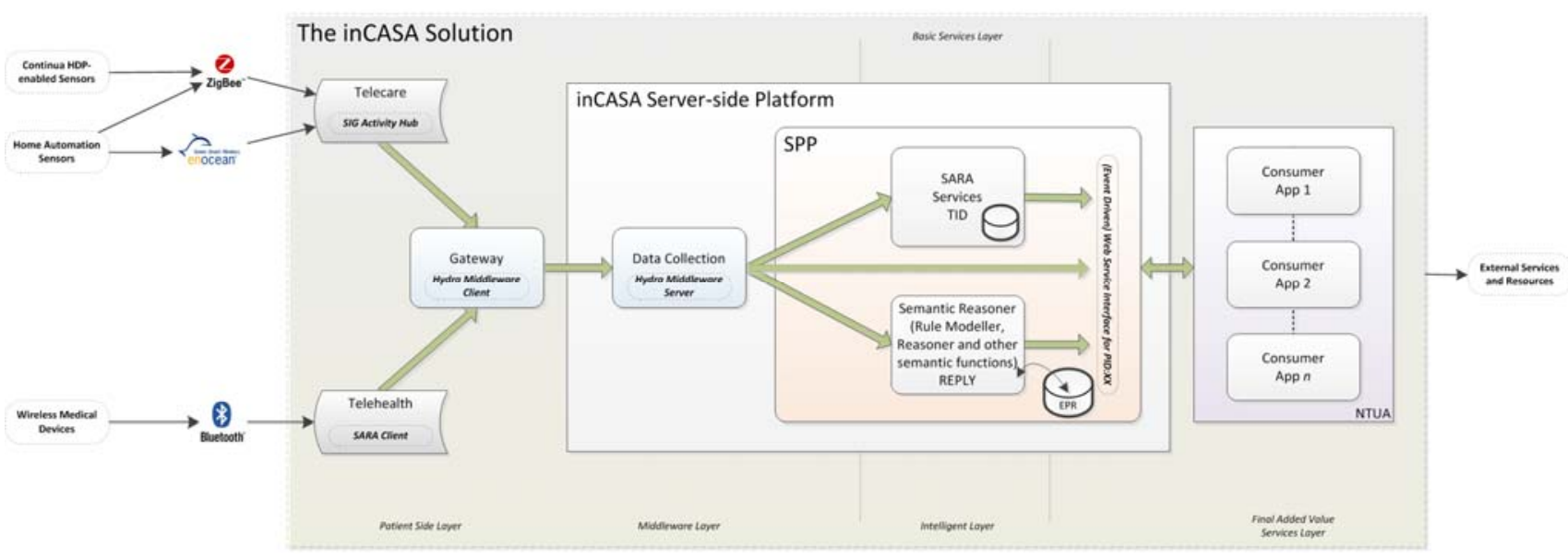

Fig. 3 - Two-level inCASA gateway architecture

\subsection{PRODUCT SELECTION}

As sensors, low-cost off-the shelf environmental ZigBee sensors are used running standardized application profiles for ZigBee Home Automation [11]. They support the full registration and binding process, as well as security. For the data collector side, Atmel BitCloud is used [12]. The embedded web server and TCP/IP-stack is available from the author's team [14]. The required gateway functionality has been developed within the project.

It was one of the preconditions of the project that the system should work with commercially available off-the-shelf sensors. As the market for monitoring sensors with open and standardized network interfaces is still in its infancy, there is only a restricted list of suppliers. Amongst those, the following have been selected and are currently applied in the project. In order to avoid misunderstanding, it should be mentioned that the selection did not include a fully systematic product scouting, but was based on objectives like availability, pricing, functionality, and support.
In the first phase, two classes of sensors are used:

- ZigBee enabled monitoring sensors from [11] for Temperature and Humidity, Window/Door, Flood Sensor, Inflammable Gas Detector, Wireless CO Detector, Wireless Liquid Petroleum Gas Detector, Activity, and Smoke Detector with Heat Sensor.

- EnOcean enabled sensors for occupancy in beds and chairs from [13]. Major additional feature of these sensors is their energy autonomy, as they run without batteries or external power supply, but make use the energy of the movement.

\subsection{Activity Hub}

Major part of the author's team is the SIG Activity Hub, which is therefore described in some more detail. It is dubbed as SIG Activity Hub and enables the connectivity between various low-end sensors (e.g. ZigBee protocol and EnOcean radio protocol) and transforms them into XML-IP-format to feed into Hydra platform. It is shown in Fig. 4.

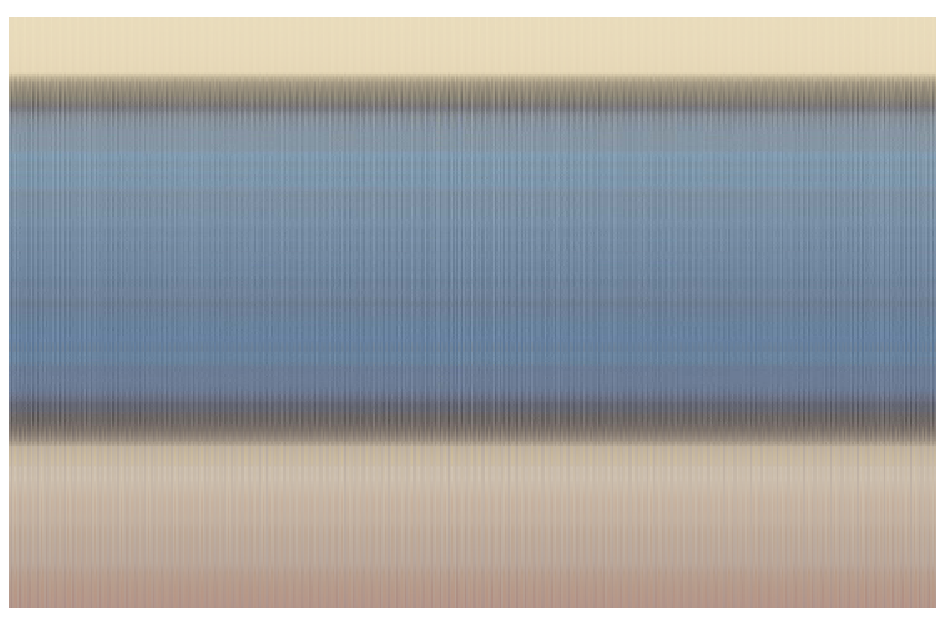

Fig. 4 - SIG activity hub in its housing 
It is anticipated in the inCASA architecture to have one SIG activity hub for each apartment. For the physical installation of the SIG activity hub, the following conditions must be met:

- For the easiest deployment mode, the activity hub will be mains powered. Therefore, an access to the electricity network must be available.

- The SIG activity hub must communicate with the wireless sensors in the apartment. This leads to a possibly central positioning in the apartment, so that a direct communication can be supported. In case of the ZigBee enabled networks, this criterion is of lesser importance as ZigBee network layer supports mesh networking. In this case, intermediate other sensor nodes could possibly forward the data to the activity hub. However, using the inCASA basic sensor set, all sensors are run as ZigBee Reduced Function Devices (RFD) only, which do not come with routing capabilities. For this purpose, a mainspowered router, like e.g. a smoke alarm, would be required.

- On the other side, the SIG activity hub shall forward the monitored data to the Hydra middleware platform. For this communication, an IP-based communication channel must be accessible. For the lower network interface layer, the SIG activity hub supports the following options: Ethernet (IEEE802.3), Wireless LAN (IEEE802.11), mobile communication (GPRS), or legacy Public Switched Telephone Network (PSTN) modems. Within the inCASA project, both Ethernet and GPRS based solutions are applied.

The Activity Hub forwards its data to the middleware via HTTP-XML. Transmissions can be both event or time driven. Actual data can also be observed on a website, hosted either locally - with the known limitations in performance - or on a portal server. In addition, the activity comes with an extensive management view (shown in Fig. 5).

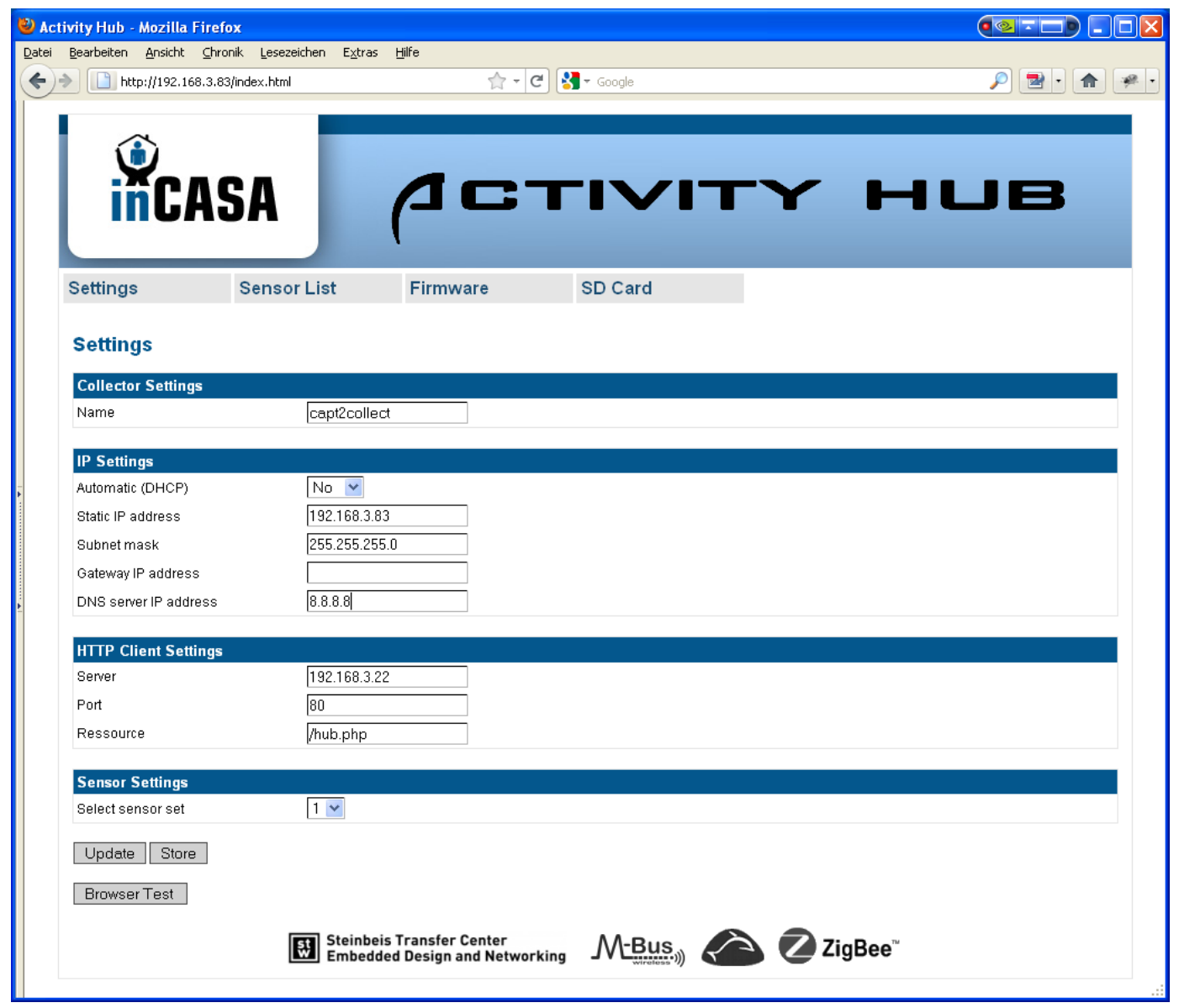

Fig. 5 - Configuration Interface of the SIG Activity hub

\section{OUTLOOK}

Two of the inCASA pilot installations will include the described elements. It will be used exclusively with the partner Agenzia Territoriale per la casa (ATC) della Provincia di Torino. ATC
Torino is the public body with responsibility for social and public housing in the area of Torino and its surroundings. The Pilot will focus on user behavior, providing a useful profile to search and determine behavior that could lead to health problems. Prepilot installations are currently already 
used for testing purposes.

In addition, it will be used together with other systems in Greece (Konstantopouleio General Hospital of Nea Ionia Agia Olga, KGHNI). Both are described in a little bit more detail in [17].

The further pilot installations of inCASA project are planned in UK (Chorleywood Health Centre), Spain (Fundación Hospital Calahorra), and France (Institut National de la Sante et de la Recherche Medicale, INSERM).

\section{ACKNOWLEDGMENT}

The inCASA project is partially funded by the European Commission under the ICT Policy Support Programme.

\section{REFERENCES}

[1] www.aal-kongress.de/

[2] www.biotronik.de/en/de/3107

[3] www.drk-koeln.de/was-wir-tun/fuer-senioren/ hausnotruf.html

[4] www.incasa-project.eu/

[5] A. Sikora, Classifications for short-range wireless networks, Wireless Congress 2005, Munich, Germany, 9-10. November 2005.

[6] D. Lill, A. Sikora, Commissioning, relaying, and monitoring of large-scale wireless metering networks, Wireless Congress, Munich, Nov. 2010.

[7] www.continuaalliance.org/index.html

[8] www.bluetooth.com/English/Experience/Pages/ Closer-Look-HDP.aspx

[9] www.zigbee.org/healthcare

[10] www.hydramiddleware.eu/

[11] www.netvox.com.tw/product.asp

[12] www.atmel.com/dyn/products/tools_card.asp? tool_id=4495\&category_id=163\& family_id=676\& subfamily_id $=2124$

[13] www.funkstuhl.de/

[14] www.stzedn.de/embetter-webserver.132.html

[15] sourceforge.net/projects/linksmart/

[16] M. Clarke et.al., Interoperable Telecare over IP - architecture, standards and Telehealth convergence, Final Version 3.3, Oct. 2011.

[17] A. Sikora, Pilot installations for combined telecare and telehealth services, 45 Jahrestagung der Deutschen Gesellschaft für Biomedizinische Technik (DGBMT), Sep. 2011, Freiburg.

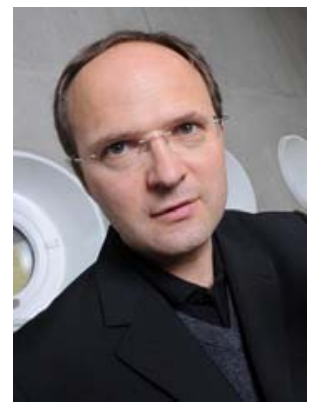

Dr.-Ing. Axel Sikora holds the Professorship of Embedded Systems and Communication Electronics at the University of Applied Sciences in Offenburg (D). He earned a Dipl.-Ing. degree in electrical engineering and a Dipl. Wirt.-Ing. degree in business administration, both from RWTH Aachen Technical

University (D). His Ph.D. was in the field of digital circuit design at Fraunhofer Institute IMS, Duisburg (D). After several positions in telecommunications and semiconductor industry, he was appointed Professor at Baden-Württemberg Cooperative State University Loerrach (D) in 1999, and joined Offenburg University in 2011.

He has founded the Steinbeis-Innovation Center Embedded Design and Networking (sizedn) in 2002, which concentrates on algorithm development, protocol development, protocol implementation, simulation and test in the field of wired and wireless embedded networking.

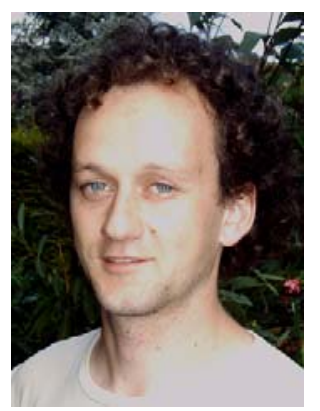

Nathan Braun holds a Dipl.Ing. degree in project management in mechatronics from University of Applied Science Basel $(\mathrm{CH})$, Université de Haute Alsace Mulhouse (F) and University of Cooperative Education Loerrach (D) in 1992. Since then, he works as software development architect at sizedn and group leader. He is mainly interested in the development and optimization of an embedded TCP/IP stack, and in the implementation of wireless sensor networks.

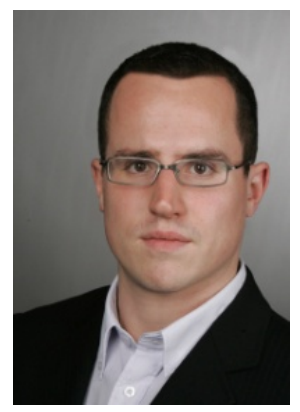

Steffen Jaeckel studied Computer Engineering at the University of Applied Sciences, Furtwangen and received his Dipl.-Inform. degree in April 2009. After that, he continued to work as project engineer at sizedn mainly in the field of embedded wireless and wired communication, embedded security and hardware design for networking protocols.

Daniel Jaeckle holds a B.Eng. in Information Technology from the Baden-Wuerttemberg Cooperative State University Loerrach (D). Besides working in the field of wireless sensor networks at sizedn, he is currently studying towards a M.Sc. in Computer Science at the University Freiburg. 